\title{
Deterministically fabricated strain-tunable quantum dot single-photon sources emitting in the telecom O-band $\odot$
}

Cite as: Appl. Phys. Lett. 117, 224001 (2020); https://doi.org/10.1063/5.0030991

Submitted: 26 September 2020 • Accepted: 10 November 2020 • Published Online: 01 December 2020

N. Srocka, P. Mrowiński, J. Große, et al.

\section{COLLECTIONS}

Paper published as part of the special topic on Non-Classical Light Emitters and Single-Photon Detectors

F This paper was selected as Featured
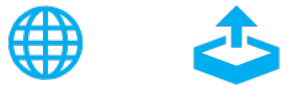

\section{ARTICLES YOU MAY BE INTERESTED IN}

Deterministically fabricated quantum dot single-photon source emitting indistinguishable photons in the telecom O-band

Applied Physics Letters 116, 231104 (2020); https://doi.org/10.1063/5.0010436

Purcell-enhanced single-photon emission from a strain-tunable quantum dot in a cavitywaveguide device

Applied Physics Letters 117, 254002 (2020); https://doi.org/10.1063/5.0033213

Progress in quantum-dot single photon sources for quantum information technologies: $\mathrm{A}$ broad spectrum overview

Applied Physics Reviews 7, 021309 (2020); https://doi.org/10.1063/5.0010193

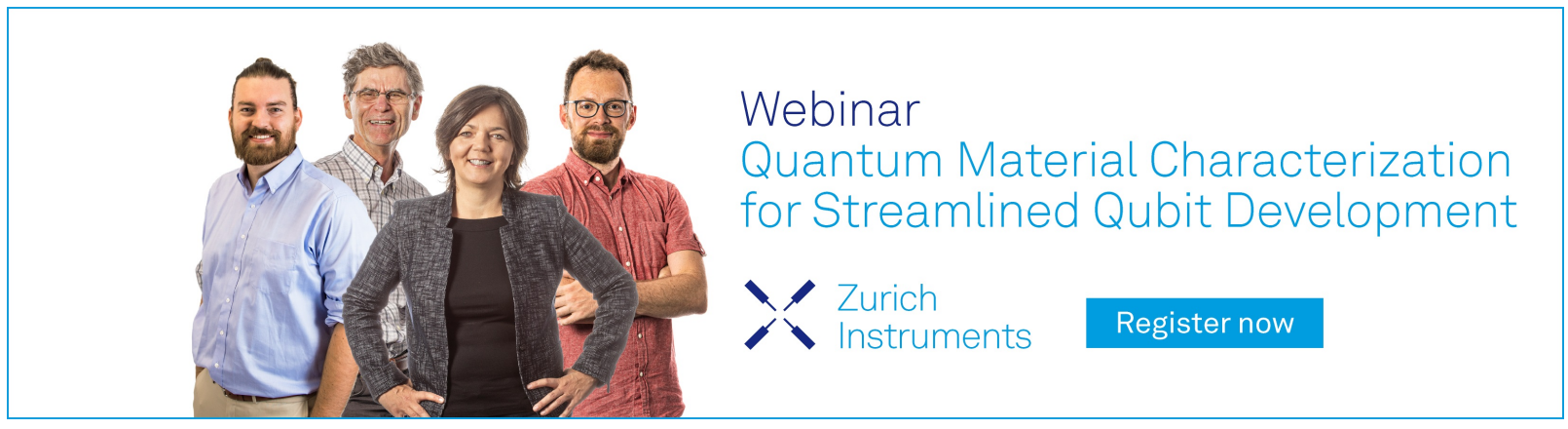




\title{
Deterministically fabricated strain-tunable quantum dot single-photon sources emitting in the telecom O-band
}

Cite as: Appl. Phys. Lett. 117, 224001 (2020); doi: 10.1063/5.0030991

Submitted: 26 September 2020 - Accepted: 10 November 2020 •

Published Online: 1 December 2020

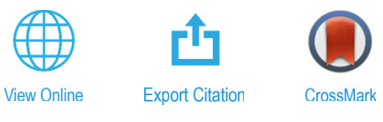

N. Srocka, ${ }^{1}$ P. Mrowiński, ${ }^{1,2}$ J. Große, ${ }^{1}$ M. Schmidt, ${ }^{1,3}$ S. Rodt, ${ }^{1}$ and S. Reitzenstein ${ }^{1, a)}$ (iD

\begin{abstract}
AFFILIATIONS
${ }^{7}$ Institut für Festkörperphysik, Technische Universität Berlin, Hardenbergstraße 36, D-10623 Berlin, Germany

${ }^{2}$ Laboratory for Optical Spectroscopy of Nanostructures, Department of Experimental Physics, Wrocław University of Technology, Wybrzeże Wyspiańskiego 27, Wrocław, Poland

${ }^{3}$ Physikalisch-Technische Bundesanstalt, Abbestraße 2-12, 10587 Berlin, Germany
\end{abstract}

Note: This paper is part of the APL Special Collection on Non-Classical Light Emitters and Single-Photon Detectors.

a) Author to whom correspondence should be addressed: stephan.reitzenstein@physik.tu-berlin.de

\begin{abstract}
Most quantum communication schemes aim at the long-distance transmission of quantum information. In the quantum repeater concept, the transmission line is subdivided into shorter links interconnected by entanglement distribution via Bell-state measurements to overcome inherent channel losses. This concept requires on-demand single-photon sources with a high degree of multi-photon suppression and high indistinguishability within each repeater node. For a successful operation of the repeater, a spectral matching of remote quantum light sources is essential. We present a spectrally tunable single-photon source emitting in the telecom O-band with the potential to function as a building block of a quantum communication network based on optical fibers. A thin membrane of GaAs embedding InGaAs quantum dots (QDs) is attached onto a piezoelectric actuator via gold thermocompression bonding. Here, the thin gold layer acts simultaneously as an electrical contact, strain transmission medium, and broadband backside mirror for the QD-micromesa. The nanofabrication of the QDmicromesa is based on in situ electron-beam lithography, which makes it possible to integrate pre-selected single QDs deterministically into the center of monolithic micromesa structures. The QD pre-selection is based on distinct single-QD properties, signal intensity, and emission energy. In combination with strain-induced fine tuning, this offers a robust method to achieve spectral resonance in the emission of remote QDs. We show that the spectral tuning has no detectable influence on the multi-photon suppression with $\mathrm{g}^{(2)}(0)$ as low as $2 \%-4 \%$ and that the emission can be stabilized to an accuracy of $4 \mu \mathrm{eV}$ using a closed-loop optical feedback.
\end{abstract}

Published under license by AIP Publishing. https://doi.org/10.1063/5.0030991

The emission of single photons with controllable wavelength and high indistinguishability is a key parameter in quantum light sources for quantum nanophotonics. It is, for example, required to implement boson sampling experiments ${ }^{1}$ and to realize entanglement swapping via Bell-state measurements in large-distance quantum communication networks based on the quantum repeater concept. ${ }^{2-4}$ In fact, in the case of the quantum repeater, it is crucial to fabricate a chain of wavelength-tunable quantum light sources that provide identical photons on demand. Even more, it is important to operate such light sources at telecommunication wavelengths, i.e., within the telecom $\mathrm{O}$ band $(\sim 1.3 \mu \mathrm{m})$ or C-band $(\sim 1.55 \mu \mathrm{m})$ with enhanced brightness ${ }^{5}$ and a deterministic fabrication scheme, ${ }^{6}$ to pave the way toward the real-world implementation of long-distance quantum communication networks via optical fibers, as recently reported using electrical Stark tuning of a quantum dot (QD) device.

In this work, we demonstrate technological advances and experimental findings to realize wavelength-tunable quantum emitters of high single-photon purity in the telecom O-band. The strain-tunable emitters are based on self-assembled InGaAs quantum dots that are deterministically integrated into photonic nanostructures attached to piezoelements by means of a flip-chip process. ${ }^{8,9}$ Here, the emission range of the InGaAs QDs is redshifted to the telecom O-band in the epitaxial growth process by using a strain-reducing layer (SRL), ${ }^{10,11}$ while the piezoelectric actuator allows us to apply an external strain field to fine tune and stabilize the QD emission wavelength during operation of the quantum light sources. To demonstrate their 
application potential, we examined the optical properties of two strain-tunable O-band single-QD mesa structures. The emission of their charged excitonic (CX) states, which are relevant for efficient single-photon generation, ${ }^{12}$ is first studied regarding multi-photon suppression. Second, we test the wavelength tuning capabilities via strain-tuning and we introduce active spectral stabilization based on a closed-loop proportional-integral-derivative (PID) controller. Finally, we demonstrate strain-tuning and active stabilization of two remote QDs, which is a crucial step over existing results toward the real-world application of QD single-photon source (SPS) in advanced quantum communication schemes where it can pave the way toward twophoton interference (TPI) of remote sources in the telecom O-band required for the implementation of fiber-based quantum repeater networks.

We manufactured our device in three main technological steps: (i) growing a semiconductor heterostructure by metal organic chemical vapor deposition, (ii) gold bonding an about $900 \mathrm{~nm}$ thick membrane of this structure onto a piezoelectric actuator, and (iii) nanostructuring the QD membrane deterministically by low-temperature cathodoluminescence (CL) scanning and in situ electron beam lithography (EBL).

First, a $200 \mathrm{~nm}$ GaAs buffer is grown on an n-doped GaAs (100) substrate followed by a $1 \mu \mathrm{m}$ thick $\mathrm{Al}_{0.90} \mathrm{Ga}_{0.10}$ As etch stop layer, a $2 \mu \mathrm{m}$ thick GaAs layer, and another $100 \mathrm{~nm}$ thick $\mathrm{Al}_{0.90} \mathrm{Ga}_{0.10}$ As layer. These three layers and the substrate are sacrificial layers to be removed during post-growth processing. The final $879 \mathrm{~nm}$ thick region of GaAs forms the active device membrane and includes a single InGaAs QD layer combined with the SRL, with the QD layer located $637 \mathrm{~nm}$ above the second etch stop layer [see Fig. 1(a)]. The QD layer is formed by 1.5 monolayers of $\operatorname{In}_{0.7} \mathrm{Ga}_{0.3}$ As followed by a 0.5 monolayer $\mathrm{GaAs}$ flush. The subsequent $5.5 \mathrm{~nm}$ thick InGaAs SRL has a gradual decrease in the In content from $30 \%$ to $10 \%$ over the first $3.5 \mathrm{~nm}$.

Next, the as-grown sample and a PIN-PMN-PT $\left[\mathrm{Pb}\left(\mathrm{In}_{1 / 2} \mathrm{Nb}_{1 / 2}\right) \mathrm{O}_{3}-\right.$ $\mathrm{Pb}\left(\mathrm{Mg}_{1 / 3} \mathrm{Nb}_{2 / 3}\right) \mathrm{O}_{3}-\mathrm{PbTiO}_{3}$ ] piezoelectric crystal are sputtered with $250 \mathrm{~nm}$ of gold. Their surfaces are then activated under argon plasma and, immediately after being removed from the deposition tool, bonded face-to-face under ambient air and thermocompression. In this step, a pressure of $6 \mathrm{MPa}$ and a temperature of about $600 \mathrm{~K}$ are applied. This process allows for a stable bond that is not

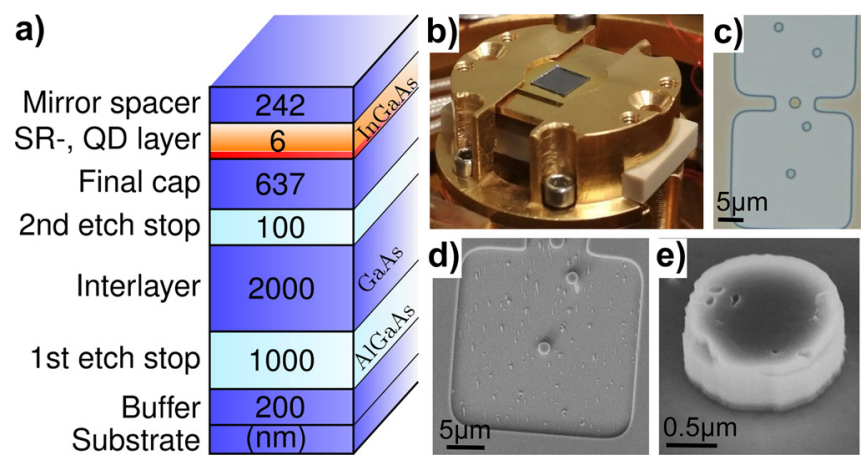

FIG. 1. (a) Sample structure as grown. (b) Sample mounted in the customized spring holder of the cryostat. (c) Optical microscope image of the sample after resist development. (d) SEM image of the processed sample showing the mapping area with the two mesas hosting QD1 and QD2. (e) SEM image of the investigated mesa hosting QD1. affected by the inherent surface roughness of the PIN-PMN-PT crystal. For more details on this process, see Ref. 13.

The flip-chip process is finalized by a layer-by-layer wet-etching process. First, the $400 \mu \mathrm{m}$ thick GaAs substrate is removed by a fast and aggressive etchant $\left(\mathrm{H}_{2} \mathrm{O}_{2} / \mathrm{NH}_{3}, 10: 1\right)$ and stopped at the first $\mathrm{Al}_{0.9} \mathrm{Ga}_{0.1}$ As layer due to the high selectivity of the wet-chemical etching process. ${ }^{14} \mathrm{HCl}$ acid is then used to solely remove the exposed etch stop layer. In our process, we use $\mathrm{HCl}$ instead of the typically applied $\mathrm{HF}$ mainly because of the lower etch rate that leads to better process control and higher quality of the etched surfaces. The now-exposed second and thinner GaAs layer $(2 \mu \mathrm{m})$ is lifted off by a slower etchant (citric acid/ $\mathrm{H}_{2} \mathrm{O}_{2}, 4: 1$ ), which leads to a significantly improved surface roughness compared to a single-step etch process. Again, the second etch stop layer is removed by HCL acid. At this point, an $885 \mathrm{~nm}$ thick membrane, containing the single QD layer, is gold bonded to a PIN-PMN-PT crystal and completely freed from sacrificial layers.

In preparation for the following main processing step, the sample is spin coated with a nominal $300 \mathrm{~nm}$ thick layer of AR-P 6200 (CSAR 62) electron-beam resist. The sample is then loaded in a special scanning electron microscope (SEM) that enables CL measurements at cryogenic temperatures. The sample is cooled to $10 \mathrm{~K}$, and the CL signal is mapped over a $20 \mu \mathrm{m} \times 20 \mu \mathrm{m}$ large area with a voxel size of $0.5 \mu \mathrm{m}$ to identify the position of bright and isolated QDs that emit at the desired wavelength in the telecom O-band. A corresponding map showing spatially resolved CL in a spectral range of (1300-1305) $\mathrm{nm}$ is shown in Fig. 2(a). By applying a 2D Gaussian fit, we can select the two circled QDs in Fig. 2(a) with a lateral accuracy of about $40 \mathrm{~nm}$. In the following electron beam lithography (EBL) step, deterministic circular mesa structures are written into the resist at low temperature $(10 \mathrm{~K})$ in the same SEM system. When the resist is developed, the image areas are cleared and only the EBL-structured mesa structures remain and form an etching mask for the final reactive ion etching in an inductively coupled plasma. For the circular QD micromesas with a gold mirror on the back, we expect a moderately high photon extraction efficiency $\eta$ in the range of $5 \%-10 \% .{ }^{13,15}$ This parameter can possibly be increased in the future by applying the present manufacturing method to sources based on circular Bragg resonators (CBRs) that promise broadband photon extraction efficiency up to $\eta=95 \%$ in the telecom O-band. ${ }^{16}$ In fact, our developed device processing is fully compatible with the realization of CBRs, and using a soon available commercial in situ EBL system, we plan to implement $1.3 \mu \mathrm{m}$ QD-CBRs. In this context, it will be interesting to explore the effect of piezostrain tuning on both the QD and cavity properties, which could probably be used to induce a piezocontrolled slight ellipticity in the CBRs to realize high-performance QD-SPS with linearly polarized emission under resonant excitation. ${ }^{17}$

Noteworthily, and in contrast to previous work on piezoelectric tuning of QD-micromesas emitting in the $930 \mathrm{~nm}$ range, ${ }^{18}$ the etching depth used in the present work corresponds to the thickness of the gold-bonded membrane in our design. Thus, the base diameter of the mesa structure is the only cross section to transfer the strain-field effect at the piezoelectric actuator to the QD position. As we discuss below, the limited contact area between the semiconductor and the piezoelectric actuator does not have a strong impact on the achievable tuning range, which is rather limited by non-ideal wafer-bonding.

The properties of the optical device are investigated by means of microphotoluminescence ( $\mu \mathrm{PL}), \mu \mathrm{PL}$-excitation ( $\mu \mathrm{PLE})$, and 

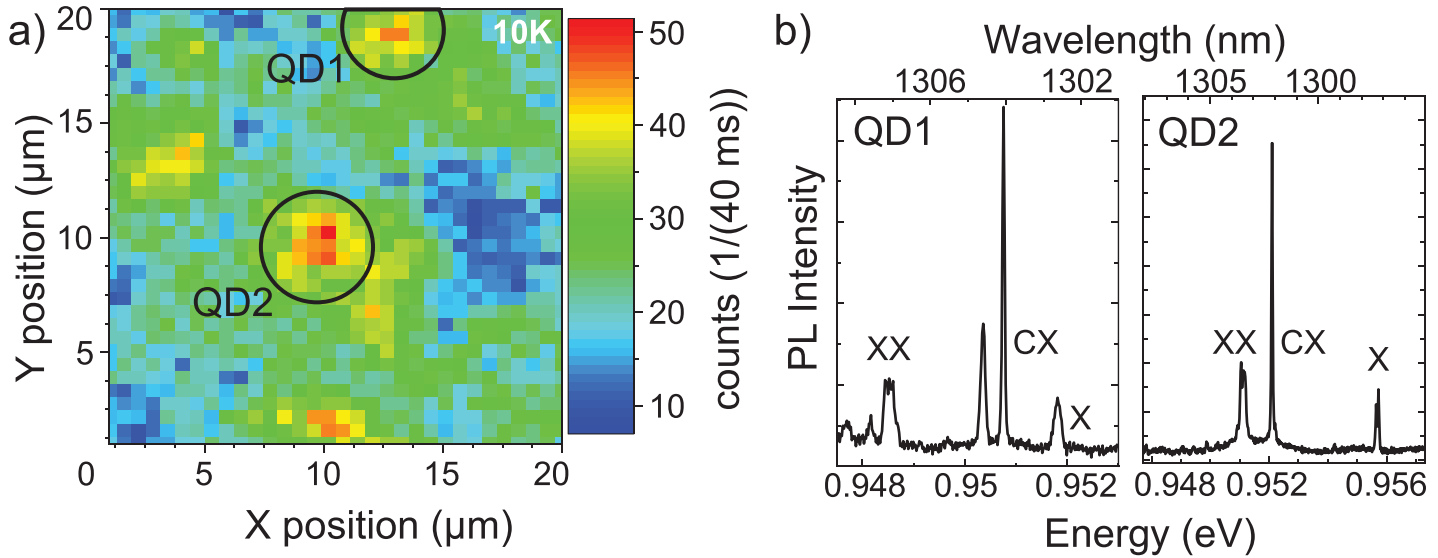

FIG. 2. (a) CL map of the pre-selection step at $10 \mathrm{~K}$ with QD1 and QD2 encircled. The spectral range of $1300 \mathrm{~nm}-1305 \mathrm{~nm}$ is depicted. (b) Corresponding $\mu \mathrm{PL}$ spectra of QD1 and QD2 after mesa processing was completed.

photon-correlation spectroscopy at $10 \mathrm{~K}$. The used helium-flow cryostat has an extra-high-voltage feedthrough to electrically connect the piezoelectric actuator to a voltage supply and a customized spring holder to facilitate strain-tuning of the QD-micromesas. The deterministic QD mesa structures are optically excited by a continuouswave $(\mathrm{cw})$ diode laser $(785 \mathrm{~nm})$ or a tunable pulsed laser providing ps-pulses at a repetition rate of $80 \mathrm{MHz}$. The photoluminescence signal is collected with an objective [numerical aperture $(\mathrm{NA})=0.4$ ] and spectrally resolved in a grating spectrometer (spectral resolution $\sim 20 \mu \mathrm{eV}$ ). The photon stream can either be detected by a liquid nitrogen cooled InGaAs-array detector or fiber-based Hanbury Brown and Twiss (HBT) and Hong-Ou-Mandel (HOM) configurations attached to the output slit of the monochromator. The quantum optical configurations include two superconducting nanowire single-photon detectors (SNSPDs) (a temporal resolution of approximately 50 ps and a detection efficiency of approximately $80 \%$ at $1310 \mathrm{~nm}$ ).

Device characterization starts by basic $\mu \mathrm{PL}$ measurements under non-resonant $\mathrm{cw}$ excitation $(785 \mathrm{~nm})$ at $10 \mathrm{~K}$. We identified two photonic structures with bright CX emission lines at $1303.98 \mathrm{~nm}$ (QD1) and $1303.78 \mathrm{~nm}$ (QD2). The spectral fingerprints of these two QDs, as shown in Fig. 2(b), have three characteristic and representative emission lines, which are identified as a neutral exciton (X), singly charged exciton (CX), and biexciton (XX) by excitation-power and polarization dependent measurements (see supplementary material Figs. S1 and S2).

To demonstrate strain-tuning and to quantify the corresponding spectral shift, the QD emission is studied depending on the applied voltage to the piezoelectric actuator. The applied voltage was varied from $-400 \mathrm{~V}$ to $+400 \mathrm{~V}$, which corresponds to an electric field $F$ of $-13.4 \mathrm{kV} \mathrm{cm}^{-1}$ to $+13.4 \mathrm{kV} \mathrm{cm}{ }^{-1}$ and results in a spectral tuning range of $0.5 \mathrm{~nm}$ for the present sample, as shown in Fig. 3 . Noteworthily, other samples showed a tuning range up to $4 \mathrm{~nm}$ when the device allows for applying higher voltages of up to $\pm 33 \mathrm{kV} \mathrm{cm}^{-1}$ (cf. Fig. S3), however, at the risk of sample damage. Comparison between these results and values reported in Ref. 18 for a noncompletely etched QD-mesa shows that the complete removal of the semiconductor material around the QD-mesa does not significantly reduce the available tuning range since in the electric field range of $\pm 20 \mathrm{kV} / \mathrm{cm}$, the achieved relative spectral shift of $(0.24 \pm 0.02) \%$ for device B presented in the supplementary material, which is consistent with $(0.20 \pm 0.02) \%$ that can be extracted for the device discussed in Ref. 18. In fact, we observe strong device-to-device variations of the available tuning range, which indicates non-ideal wafer-bonding using our home-made bonding tool, and we expect higher tuning ranges and better reproducibility by using a commercial wafer bonder in the future.

In addition to the desired wavelength shift of our piezocontrolled QD-micromesas, we observe spectral fluctuation over time due to piezoelectric creep, which is most pronounced directly after a voltage change. To illustrate this point, Fig. 4(a) shows a systematic wavelength change of about $0.05 \mathrm{~nm}$ over the first 5 min after setting the piezovoltage. The creep effect decreases significantly after $30 \mathrm{~min}$ but does not vanish completely over time. This behavior is similar to

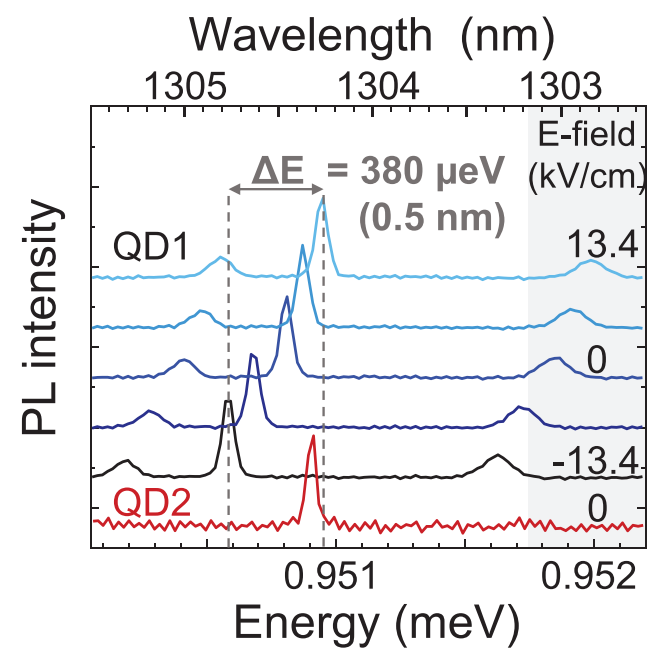

FIG. 3. Energy tuning of the CX emission line of QD1 (black to blue). An energy shift of $380 \mu \mathrm{eV}(0.5 \mathrm{~nm})$ was observed when changing the electric field applied to the piezoelectric actuator from $-13.4 \mathrm{kV} / \mathrm{cm}$ to $+13.4 \mathrm{kV} / \mathrm{cm}$. The CX emission line of QD2 without the applied electric field (red) is within the energetic tuning limits of QD1. 
a)

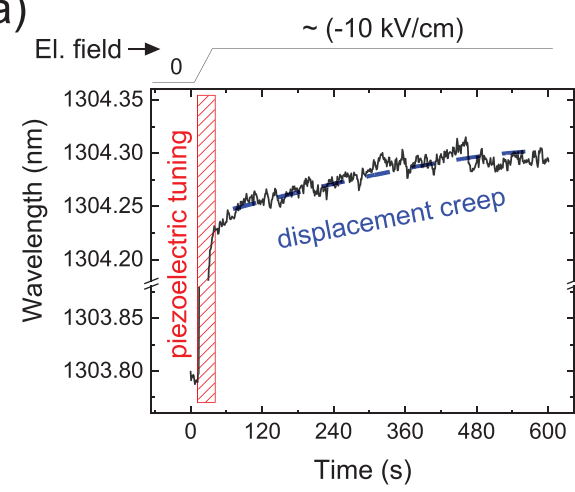

b)

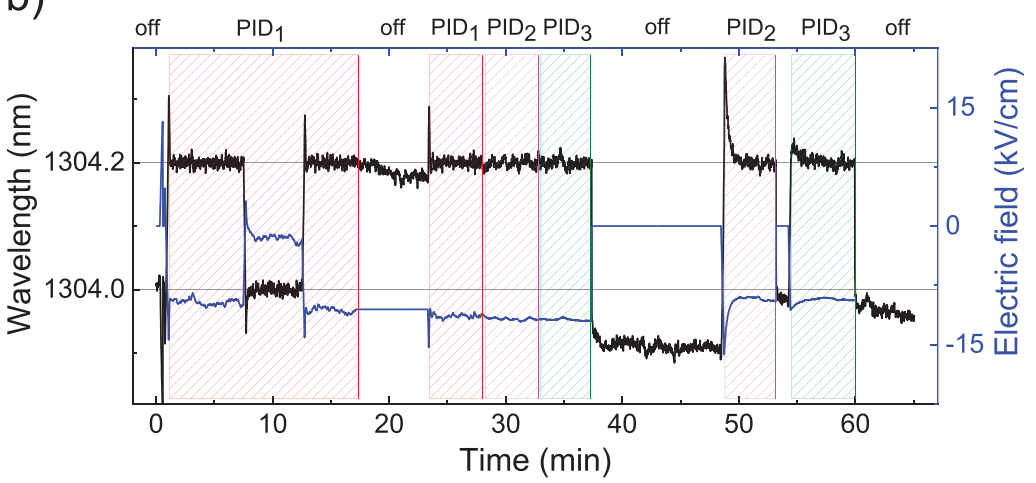

FIG. 4. (a) Impact of the piezoelectric actuator's creep on the wavelength of the CX emission line of QD1 over time. (b) PID stabilization tests on QD1 (see Table I for the summary).

earlier observations in piezocontrolled QD devices. ${ }^{8,18,19}$ In addition, we examined the relative change of the emission energies of $\mathrm{X}, \mathrm{XX}$, and $\mathrm{CX}$ and we found that the binding energy is only slightly influenced, on a scale of $0.3 \mathrm{meV}$, by the applied strain (see supplementary material Fig. S3). This change in binding energy is significantly smaller than values on the order of meV observed for planar QD samples with obviously better strain transfer. ${ }^{20}$ For some QDs, the excitonic fine structure splitting (FSS) could be reduced from $40 \mu \mathrm{eV}$ to $20 \mu \mathrm{eV}$ [see supplementary material Fig. S3(d)]. Further FSS reduction is not possible in our case. In fact, a second degree of freedom in the piezoelectric actuator would be required to fully symmetrize the distorted confinement potential, being responsible for the FSS. ${ }^{21}$

Long-term spectral stability is one of the most important aspects of a practical single-photon source for quantum communication applications based, e.g., on entanglement swapping via Bell-state measurements. Therefore, the effect of creep on the wavelength stability is an important issue to address. One can efficiently stabilize the emission wavelength, as it was reported for a quantum light emitting diode ${ }^{8}$ and later also realized for a deterministically fabricated InAs/GaAs QD microlens, ${ }^{18}$ by using an active optical feedback (closed-loop) algorithm. In the implemented concept, the emission wavelength is monitored by Lorentzian fitting of $\mu$ PL spectra taken regularly within an integration time of $0.2-1.0 \mathrm{~s}$, depending on the signal intensity. The center position of the fit is compared with the target wavelength, and the determined deviation initiates a re-adjustment procedure of the input voltage to shift the emission line back to the set target value. This re-adjustment is based on a PID algorithm.

The implemented wavelength stabilization is demonstrated for the CX emission of QD1 for several PID settings. In this test, we set the proportional gain (PG) to 100 , and the integral time (IT) parameter to $0.01 \mathrm{~s}, 0.05 \mathrm{~s}$, and $0.1 \mathrm{~s}$ to study its impact on the QD wavelength control after a voltage change applied to the piezoactuator. A graphical representation of the associated results is shown in Fig. 4(b), where the following settings are characterized within a time window of $5 \mathrm{~min}$ for each setting. The corresponding parameter set and achieved wavelength stability are shown in Table I. Evaluation of the recorded $\mu \mathrm{PL}$ time traces shows that the best spectral stabilization with a standard deviation of the emission wavelength (energy) of $5.6 \mathrm{pm}(4.1 \mu \mathrm{eV})$ could be achieved for $\mathrm{PG}=100$ and $\mathrm{IT}=0.01 \mathrm{~s}$. Moreover, we found that by increasing PG, the stabilization time decreases. However, in order to maintain the stabilization quality, the IT value must be increased proportionally. We did not observe any clear dependence of the QD spectral stability on the change in the derivative term of the PID controller.

Next, we demonstrate resonance tuning and stabilization of CX emission lines of the two different QD-micromesas (QD1 and QD2) by applying the optimized PID settings determined before. The time evolution of the piezoelectrically stabilized CX emission wavelength is presented in Fig. 5(a), showing standard deviations of $12 \mu \mathrm{eV}$ for QD1 and $6 \mu \mathrm{eV}$ for QD2, respectively. In Fig. 5(b), we present $\mu \mathrm{PL}$ spectra of both QDs tuned into resonance and being stabilized at $1304.05 \mathrm{~nm}$ with respect to the CX emission line. The good spectral overlap of the inhomogeneously (by spectral diffusion) broadened emission lines of QD1 and QD2 under active PID control reflects the efficient fine tuning and spectral control of the QDs via the PID controlled piezoactuator. Ultimately, it has to be demonstrated that a high spectral overlap can also be achieved on a scale of the homogenous linewidth, which can be probed by HOM experiments on remote sources.

To verify the quantum nature of emission, we measured the photon-autocorrelation of the spectrally tunable CX lines of QD1 and QD2. Here, the CX transition is driven at saturation under pulsed non-resonant excitation with a repetition rate of $80 \mathrm{MHz}$. In Fig. 6, we present histograms of the correlation events recorded in the HBT configuration representing the second-order autocorrelation function $g^{(2)}(\tau)$, which is used to evaluate the multi-photon suppression at zero time delay. First, the autocorrelation functions of QD1 and QD2 were measured without the influence of the piezoinduced strain-field emitting at different wavelengths, as shown in the upper panels of Fig. 6. The measurements show pronounced antibunching for the central

TABLE I. Quantified results of the emission energy stability for various PID integral times evaluated in terms of the standard deviation of emission energy and stabilization time required for the adjustment.

\begin{tabular}{lccc}
\hline \hline PID settings (prop. gain =100) & $\mathrm{PID}_{1}$ & $\mathrm{PID}_{2}$ & $\mathrm{PID}_{3}$ \\
\hline PID integral time (s) & 0.01 & 0.05 & 0.1 \\
Standard deviation $(\mu \mathrm{eV})$ & 4.12 & 4.45 & 5.26 \\
Stabilization time $(\mathrm{s})$ & 30 & 50 & 80 \\
\hline \hline
\end{tabular}


a)

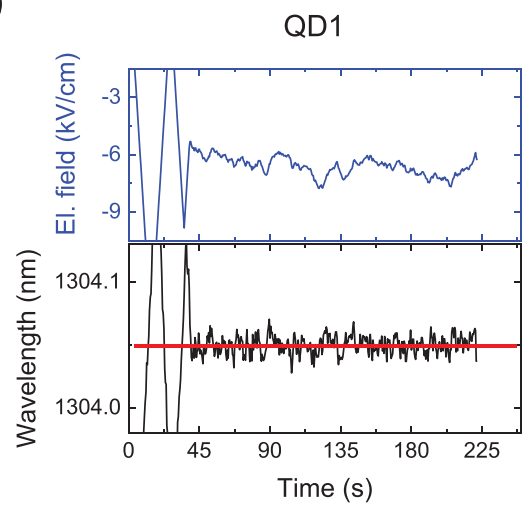

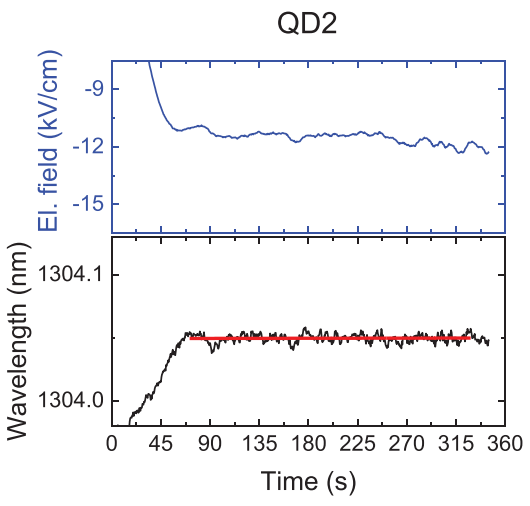

b)

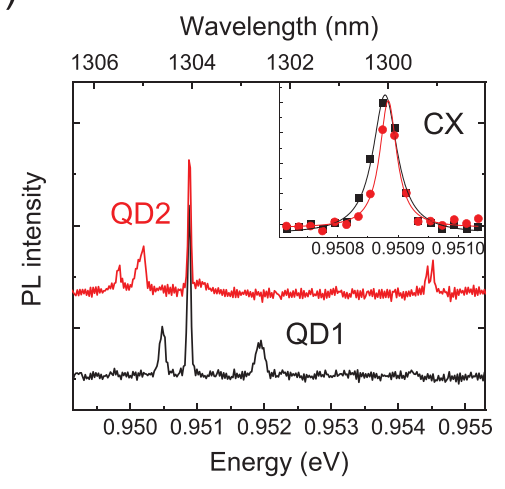

FIG. 5. (a) Emission wavelength traces of QD1 and QD2 over time to quantify the stabilization and (b) $\mu \mathrm{PL}$ spectra when both QDs are tuned in resonance via the applied piezoelectric strain field.

peak at $\tau=0 \mathrm{~ns}$, and the evaluation of the experimental data with twosided mono-exponential fit functions convoluted with the timing response of $50 \mathrm{ps}$ of the SNSPDs used in this experiment yields $g^{(2)}(0)_{f i t}=0.020_{-0.020}^{+0.030}$ for QD1 and $g^{(2)}(0)_{f i t}=0.052 \pm 0.030$ for QD2. When the electric field is applied to the piezoactuator and both QDs' CX emissions are set to the resonant wavelength and stabilized by PID for several minutes at $1304.05 \mathrm{~nm}$ (the measurements were taken independently), the antibunching behavior is preserved and the corresponding fits yield $g^{(2)}(0)_{\text {fit }}=0.040 \pm 0.030$ for QD1 and $g^{(2)}(0)_{\text {fit }}=0.053 \pm 0.030$ for QD2, confirming that the spectral tuning has no significant impact on the single-photon purity of the QD-micromesas. The achieved results are very promising with regard to the target applications requiring the emission of indistinguishable single photons from remote quantum light sources. However, we would like to point out that the photon indistinguishability of these QD-micromesas is not yet high enough for this purpose. In fact, we a)
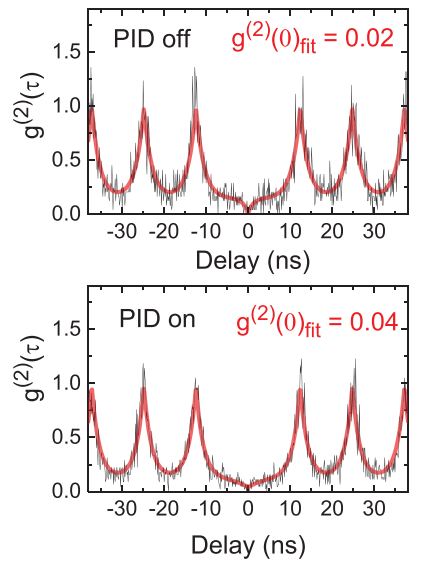

b)
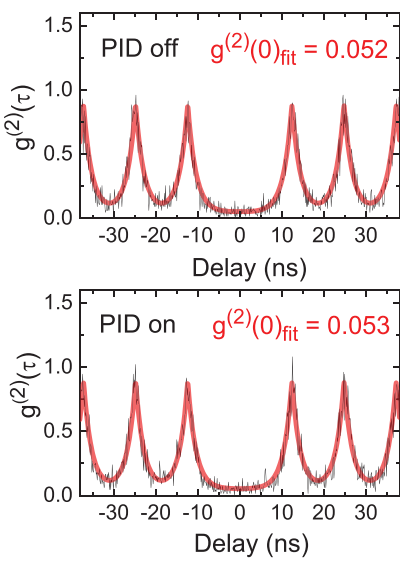

FIG. 6. (a) Autocorrelation experiments performed on the charged excitons of QD1 and (b) QD2 in the case of "on" and "off" PID controllers, which are used for stabilizing the piezocontrolled wavelength of the emitters. examined the TPI of tunable and stabilized CX emission of QD1 under pulsed p-shell excitation in the Hong-Ou-Mandel configuration. As we discuss in the supplementary material, this experiment yields a HOM visibility of $(16 \pm 8) \%$, a post-selected visibility of $(79 \pm 15) \%$, with a corresponding coherence time of $(470 \pm 85) \mathrm{ps}$, and a lifetime of $\tau_{1}=(1.54 \pm 0.05)$ ns (see the supplementary material, Figs. 5 and $6)$. These values are in agreement with results achieved recently for non-tunable QD-micromesas, ${ }^{13}$ which indicates that the optical quality and coherence of the $1.3 \mu \mathrm{m}$ QD have to be improved in the future, e.g., by optimizing the SRL, in order to fully exploit their potential in advanced quantum communication applications. For instance, the gradual increase in the In content in the SRL and the overall thickness of the SRL could be varied as an optimization parameter to achieve a smoother transition from the strongly lattice mismatched $\operatorname{In}_{0.7} \mathrm{Ga}_{0.3} \mathrm{As}$ QD layer to the GaAs matrix, thereby reducing the density of (charge trapping) defect states. Alternatively, one could also consider slowing down the GaAs growth on top of the SRL and try to better "heal" out the roughness introduced by the In-containing layers. HOM measurements can be a sensitive tool to evaluate the impact of design modifications on the optical and quantum optical properties of the $1.3 \mu \mathrm{m}$ QDs.

In summary, we demonstrated a deterministically fabricated and tunable QD single-photon source emitting in the telecommunication O-band at $1.3 \mu \mathrm{m}$. Our device consisting of a QD micromesa and attached to a piezoelectric actuator allows us to fine tune the QD's emission wavelength by up to $0.5 \mathrm{~nm}$ via a voltagecontrolled strain field. The tuning range and an implemented closed-loop PID control system enabled us to stabilize the emission energy with an accuracy (standard deviation) of up to $\sim 4 \mu \mathrm{eV}$ and to hold emission lines of two remote QD-micromesas with multiphoton-suppression better than $5 \%$ in resonance with excellent spectral overlap. These results are very promising for the future implementation of long-distance quantum communication networks.

See the supplementary material for information on the identification of excitonic complexes in QD1 and QD2, Hong-Ou-Mandel studies on the photon indistinguishability of QD1, and on optical properties of a second device with a larger strain-tuning range. 
This work was funded by the FI-SEQUR project jointly financed by the European Regional Development Fund (EFRE) of the European Union in the framework of the programme to promote research, innovation, and technologies (Pro FIT) in Germany within the 2nd Poland-Berlin Photonics Programme, Grant No. 2/POLBER-2/2016. Support from the German Research Foundation through CRC 787 "Semiconductor Nanophotonics: Materials, Models, Devices" and the Volkswagen Foundation via project "NeuroQNet" is also acknowledged. P.M. gratefully acknowledges the financial support from the Polish Ministry of Science and Higher Education within the "Mobilnosc Plus-Vedycja" program and from the Polish National Agency for Academic Exchange (NAWA) via project PPI/APM/2018/ 1/00031/U/001.

We thank T. Heindel for technical support.

\section{DATA AVAILABILITY}

The data that support the findings of this study are available from the corresponding author upon reasonable request.

\section{REFERENCES}

${ }^{1}$ H. Wang, J. Qin, X. Ding, M. C. Chen, S. Chen, X. You, Y. M. He, X. Jiang, L. You, Z. Wang, C. Schneider, J. J. Renema, S. Höfling, C. Y. Lu, and J. W. Pan, Phys. Rev. Lett. 123, 250503 (2019).

${ }^{2}$ P. Kok, C. P. Williams, and J. P. Dowling, Phys. Rev. A 68, 022301 (2003).

${ }^{3}$ H. J. Kimble, Nature 453, 1023 (2008).

${ }^{4}$ K. Azuma, K. Tamaki, and H.-K. Lo, Nat. Commun. 6, 6787 (2015).

${ }^{5}$ J. Yang, C. Nawrath, R. Keil, R. Joos, X. Zhang, B. Höfer, Y. Chen, M. Zopf, M. Jetter, S. L. Portalupi, F. Ding, P. Michler, and O. G. Schmidt, Opt. Express 28, 19457 (2020).

${ }^{6}$ M. Sartison, L. Engel, S. Kolatschek, F. Olbrich, C. Nawrath, S. Hepp, M. Jetter, P. Michler, and S. L. Portalupi, Appl. Phys. Lett. 113, 032103 (2018).

${ }^{7}$ Z. H. Xiang, J. Huwer, J. Skiba-Szymanska, R. M. Stevenson, D. J. P. Ellis, I. Farrer, M. B. Ward, D. A. Ritchie, and A. J. Shields, Commun. Phys. 3, 121 (2020).
${ }^{8}$ R. Trotta, P. Atkinson, J. D. Plumhof, E. Zallo, R. O. Rezaev, S. Kumar, S. Baunack, J. R. Schröter, A. Rastelli, and O. G. Schmidt, Adv. Mater. 24, 2668 (2012).

${ }^{9}$ B. Höfer, F. Olbrich, J. Kettler, M. Paul, J. Höschele, M. Jetter, S. L. Portalupi, F. Ding, P. Michler, and O. G. Schmidt, AIP Adv. 9, 085112 (2019).

${ }^{10}$ V. M. Ustinov, N. A. Maleev, A. E. Zhukov, A. R. Kovsh, A. Y. Egorov, A. V. Lunev, B. V. Volovik, I. L. Krestnikov, Y. G. Musikhin, N. A. Bert, P. S. Kop'ev, Z. I. Alferov, N. N. Ledentsov, and D. Bimberg, Appl. Phys. Lett. 74, 2815 (1999).

${ }^{11}$ M. Paul, F. Olbrich, J. Höschele, S. Schreier, J. Kettler, S. L. Portalupi, M. Jetter, and P. Michler, Appl. Phys. Lett. 111, 033102 (2017).

${ }^{12}$ S. Strauf, N. G. Stoltz, M. T. Rakher, L. a Coldren, P. M. Petroff, and D. Bouwmeester, Nat. Photonics 1, 704 (2007).

${ }^{13}$ N. Srocka, P. Mrowiński, J. Große, M. von Helversen, T. Heindel, S. Rodt, and S. Reitzenstein, Appl. Phys. Lett. 116, 231104 (2020).

${ }^{14}$ A. R. Clawson, Mater. Sci. Eng. R: Rep. 31, 1 (2001).

${ }^{15}$ N. Srocka, A. Musiał, P.-I. Schneider, P. Mrowiński, P. Holewa, S. Burger, D. Quandt, A. Strittmatter, S. Rodt, S. Reitzenstein, and G. Sek, AIP Adv. 8, 085205 (2018).

${ }^{16}$ L. Rickert, T. Kupko, S. Rodt, S. Reitzenstein, and T. Heindel, Opt. Express 27, 36824 (2019).

${ }^{17}$ H. Wang, Y. M. He, T. H. Chung, H. Hu, Y. Yu, S. Chen, X. Ding, M. C. Chen, J. Qin, X. Yang, R. Z. Liu, Z. C. Duan, J. P. Li, S. Gerhardt, K. Winkler, J. Jurkat, L. J. Wang, N. Gregersen, Y. H. Huo, Q. Dai, S. Yu, S. Höfling, C. Y. Lu, and J. W. Pan, Nat. Photonics 13, 770 (2019).

${ }^{18}$ M. Schmidt, M. V. Helversen, S. Fischbach, A. Kaganskiy, R. Schmidt, A. Schliwa, T. Heindel, S. Rodt, and S. Reitzenstein, Opt. Mater. Express 10, 76 (2020).

${ }^{19}$ T. Zander, A. Herklotz, S. Kiravittaya, M. Benyoucef, F. Ding, P. Atkinson, S. Kumar, J. D. Plumhof, K. Dörr, A. Rastelli, and O. G. Schmidt, Opt. Express 17, 22452 (2009).

${ }^{20}$ F. Ding, R. Singh, J. D. Plumhof, T. Zander, V. Křápek, Y. H. Chen, M. Benyoucef, V. Zwiller, K. Dörr, G. Bester, A. Rastelli, and O. G. Schmidt, Phys. Rev. Lett. 104, 067405 (2010).

${ }^{21}$ R. Trotta, J. Martín-Sánchez, J. S. Wildmann, G. Piredda, M. Reindl, C. Schimpf, E. Zallo, S. Stroj, J. Edlinger, and A. Rastelli, Nat. Commun. 7, 10375 (2016). 